\title{
Review Article \\ On-Chip All-Optical Switching and Memory by Silicon Photonic Crystal Nanocavities
}

\author{
Masaya Notomi, Takasumi Tanabe, Akihiko Shinya, Eiichi Kuramochi, and Hideaki Taniyama \\ NTT Basic Research Laboratories, NTT Corporation, 3-1 Morinosato-Wakamiya, Atsugi 2430198, Japan \\ Correspondence should be addressed to Masaya Notomi, notomi@will.brl.ntt.co.jp
}

Received 23 December 2007; Accepted 13 April 2008

Recommended by D. Lockwood

\begin{abstract}
We review our recent studies on all-optical switching and memory operations based on thermo-optic and carrier-plasma nonlinearities both induced by two-photon absorption in silicon photonic crystal nanocavities. Owing to high- $Q$ and small volume of these photonic crystal cavities, we have demonstrated that the switching power can be largely reduced. In addition, we demonstrate that the switching time is also reduced in nanocavity devices because of their short diffusion time. These features are important for all-optical nonlinear processing in silicon photonics technologies, since silicon is not an efficient optical nonlinear material. We discuss the effect of the carrier diffusion process in our devices, and demonstrate improvement in terms of the response speed by employing ion-implantation process. Finally, we show that coupled bistable devices lead to all-optical logic, such as flip-flop operation. These results indicate that a nanocavity-based photonic crystal platform on a silicon chip may be a promising candidate for future on-chip all-optical information processing in a largely integrated fashion.
\end{abstract}

Copyright (C) 2008 Masaya Notomi et al. This is an open access article distributed under the Creative Commons Attribution License, which permits unrestricted use, distribution, and reproduction in any medium, provided the original work is properly cited.

\section{INTRODUCTION}

It used to be a great challenge to tightly confine light in a wavelength-scale volume, which had limited the capability of photonics technologies in various aspects. Recently, however, an optical resonator with ultrahigh- $Q(\sim 1$ million $)$ and small volume $\left(\sim(\lambda / n)^{3}\right)$ becomes possible by employing the state-of-the-art photonic crystal technologies [1-6]. Figure 1 shows an example of high- $Q$ nanocavities, which we have recently demonstrated in silicon photonic crystals. This particular cavity shows a theoretical $Q$ of over $10^{8}$ and an experimental $Q$ of 1.3 million with a mode volume of $1.5(\lambda / n)^{3}[4,7]$. These values are hardly available in optical systems other than photonic crystals. Various forms of light-matter interactions are expected to be enhanced in such high- $Q$ nanocavities having large $Q / V$ ratio [8]. Among them, in this article, we focus on application to alloptical switching and memory operations based on optical nonlinear interaction. Especially for this, we investigate alloptical operations based on carrier-induced nonlinearity and examine the features of photonic crystal nanocavities for such applications.
As has been studied in various forms, all-optical switches can be realized using optical resonators, where a control optical pulse induces a resonance shift via optical nonlinear effects. For such a resonator-based switch, there is a twofold enhancement in terms of the switching power if a small cavity with a high- $Q$ is employed. First, the light intensity inside the cavity should be proportional to $Q / V$. Second, the required wavelength shift is proportional to $1 / Q$. In total, the switching power should be reduced by $\left(Q^{2} / V\right)$, which can be significantly large for photonic crystal nanocavities [9]. Although the switching mechanism itself is basically similar to that of previous resonatorbased switches, such as nonlinear etalons [10], this large enhancement has had an important impact on optical integration since most optical switching components require too much power for realistic integration. Furthermore, in the case of carrier-induced nonlinearity or thermo-optic nonlinearity, the smallness of the device naturally leads to fast operation speed, since the relaxation time of such processes normally depends on the size. As we will show below, this advantage is significant for wavelength-sized cavities. 


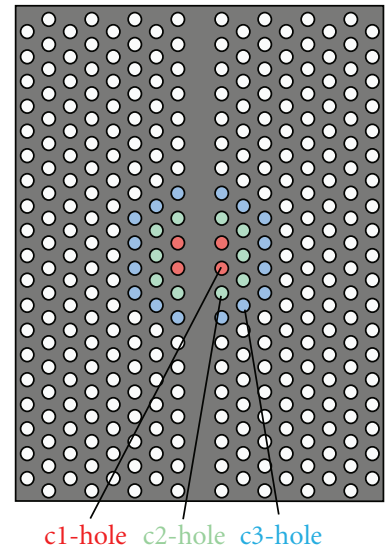

(a)

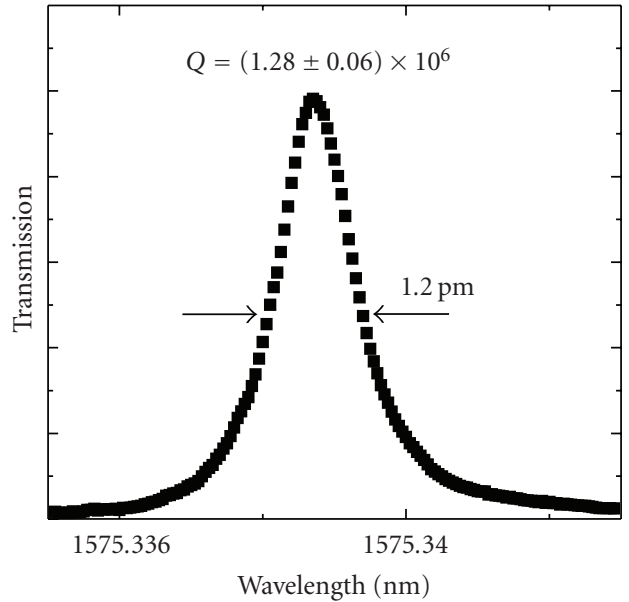

(b)

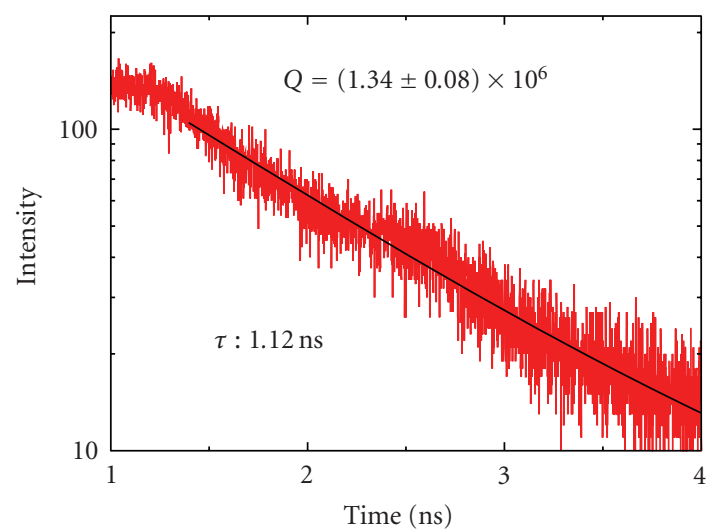

(c)

FIGURE 1: Width-modulated, line-defect photonic crystal cavities. (a) Cavity design. The cavity has the theoretical Q. The hole shifts are typically $9 \mathrm{~nm}$ (red holes), $6 \mathrm{~nm}$ (green holes), and $3 \mathrm{~nm}$ (blue holes). (b) Spectral measurement of a nanocavity fabricated in a silicon hexagonal air-hole photonic slab with $a=420 \mathrm{~nm}$ and $2 r=216 \mathrm{~nm}$. The transmission spectrum of a cavity with a second-stage hole-shift. The inner and outer hole-shifts are 8 and $4 \mathrm{~nm}$, respectively. (c) Time-domain ring-down measurement. The time decay of the output light intensity from the same cavity as (b). Details can be found in the reference.

In addition, resonator-based optical switches are well known to exhibit optical bistability [10] and thus they can be used for optical memory and all-optical logic [11]. Such functionality is one of the most important functions missing from existing photonic devices. Thus, we believe that alloptical bistable switches based on photonic crystal cavities are important candidates for future optical integration.

In this paper, we investigate all-optical switching and memory action in silicon photonic crystal nanocavity devices. First, we study the switching action using thermooptic nonlinearity. Next, we investigate similar switching action using much faster carrier-plasma nonlinearity. Thirdly, we analyze the effect of carrier relaxation process in the switching action and made an attempt to further decrease the relaxation time by ion implantation. Furthermore, we demonstrate bistable memory action employing basically the same nanocavity devices and present an example of design for on-chip all-optical logic circuits consisting of two bistable nanocavities.

\section{ALL-OPTICAL BISTABLE SWITCHING BY THERMO-OPTIC NONLINEARITY}

As described above, photonic crystal nanocavities have a promising potential for all-optical switching applications. To experimentally confirm this, we have investigated alloptical bistable switching operations employing the thermooptic nonlinearity induced by two-photon absorption (TPA) in silicon [12]. For this study, we employed an endhole shifted four-point Si photonic crystal cavity integrated with input/output photonic crystal waveguides (shown in Figure 2(a)) [13, 14]. Figure 2(b) shows transmission spectra with various input power at $1.5 \mu \mathrm{m}$ wavelength region. As clearly seen in the graph, the transmission spectrum is shifted to longer wavelength (red-shifted). This shift is due to thermo-optic nonlinearity induced by two-photon absorption in the cavity. At input power higher than $10 \mu \mathrm{W}$, there is an abrupt kink in the spectrum. This kink corresponds to bistable switching. To see this more 


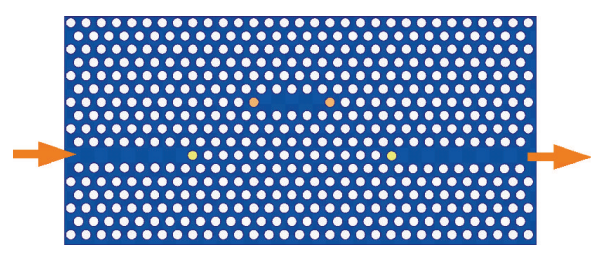

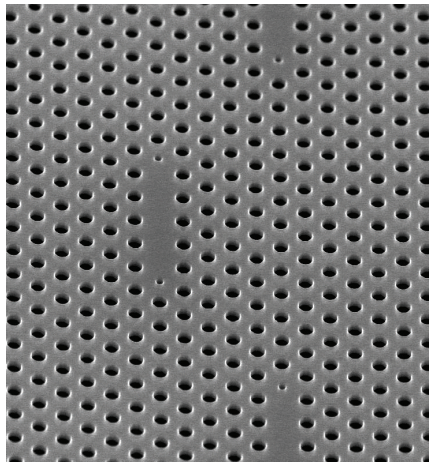

(a)

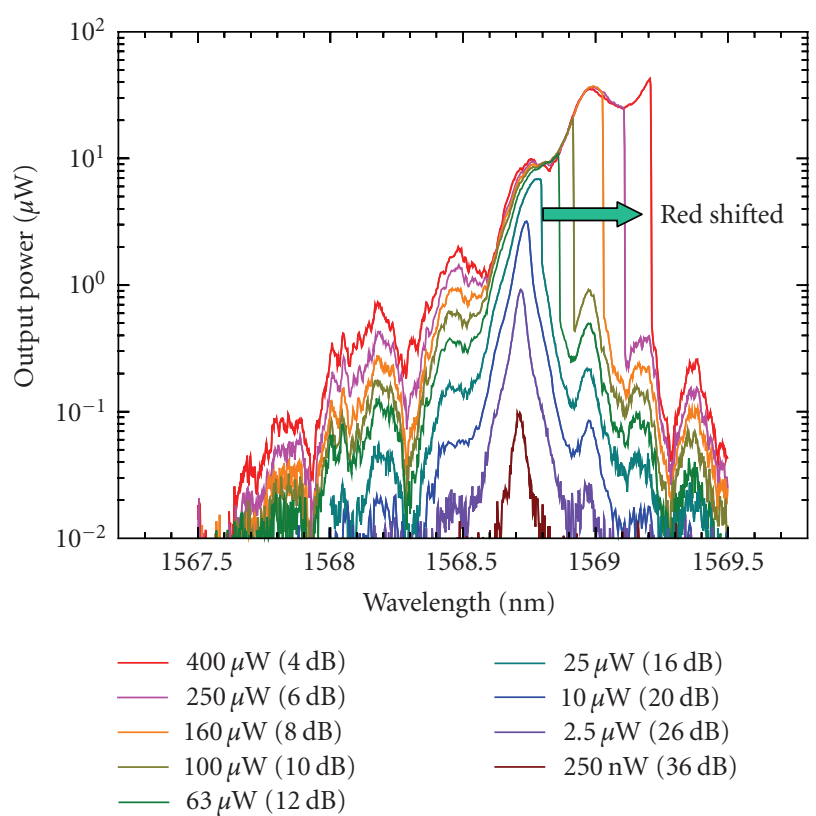

(b)

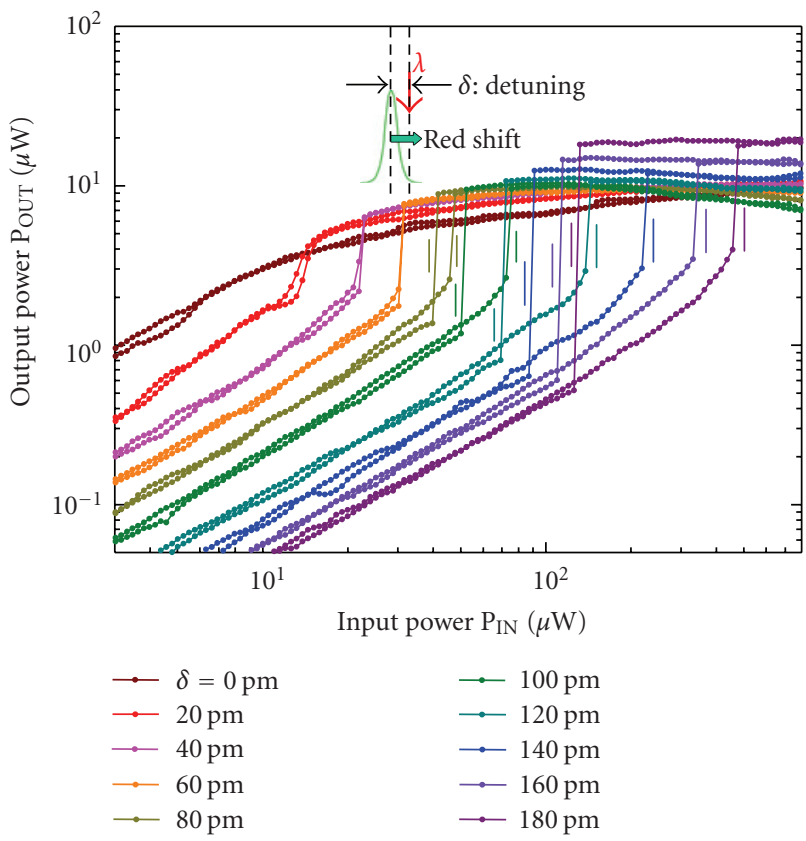

(c)

FIGURE 2: All-optical bistable switching in a silicon hexagonal air-hole photonic crystal nanocavity (end-hole shifted four-point defect cavity) realized by the thermo-optic nonlinearity induced by two-photon absorption in silicon. (a) Schematic of the sample and a scanning electron micrograph of the sample. $a=420 \mathrm{~nm}, 2 r=0.55 a$. The radius of end-holes of the cavity is $0.125 a . V=0.102 \mu \mathrm{m}^{3}$. The radius of end-holes of the waveguide is $0.15 a$. (b) Intensity-dependent transmission spectra taken by a tunable laser in the upsweep condition. $Q$ in the linear regime is 33400. (c) Output power versus input power for various detuning values. The nonlinear regime starts from $10 \mu \mathrm{W}$, and the bistable regime starts from $40 \mu \mathrm{W}$.

directly, we measured the output power as a function of the input power at various detuning conditions, as shown in Figure 2(c). Now, it is clear that this device exhibits bistable switching. The most noteworthy point regarding this switching is its switching power, which is as small as $40 \mu \mathrm{W}$.
This value is remarkably smaller that of bulk-type thermooptic nonlinear etalons (a few to several tens $\mathrm{mW}$ ) [15] and also smaller than that of recent miniature-sized thermooptic silicon microring resonator devices $(\sim 0.8 \mathrm{~mW})$ [16]. In addition, it is important to note that TPA occurs only in the 


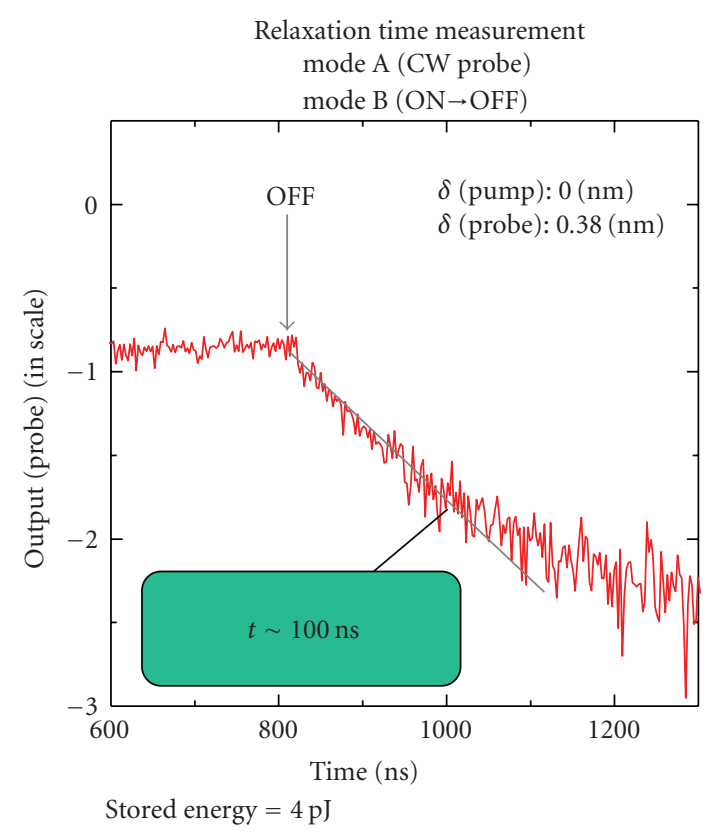

(a)

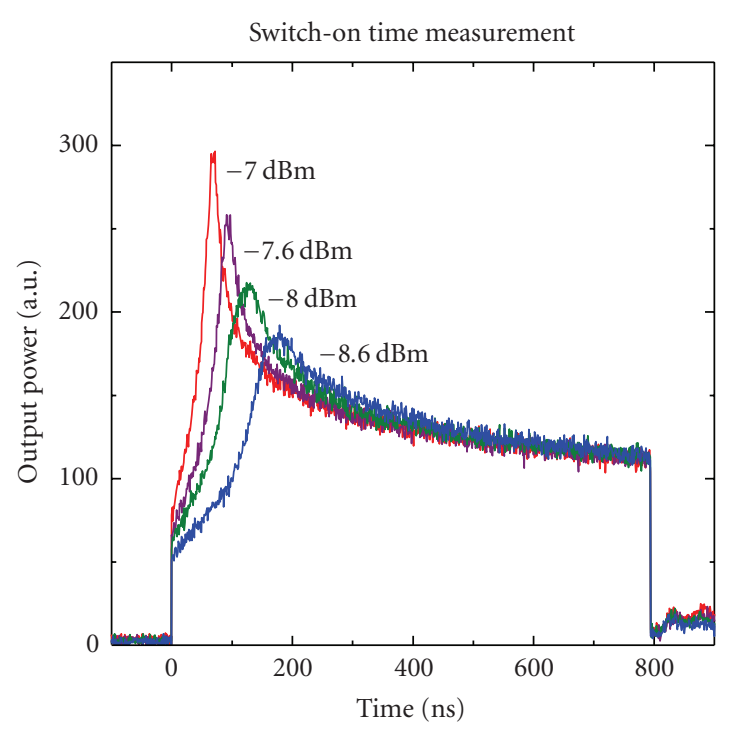

(b)

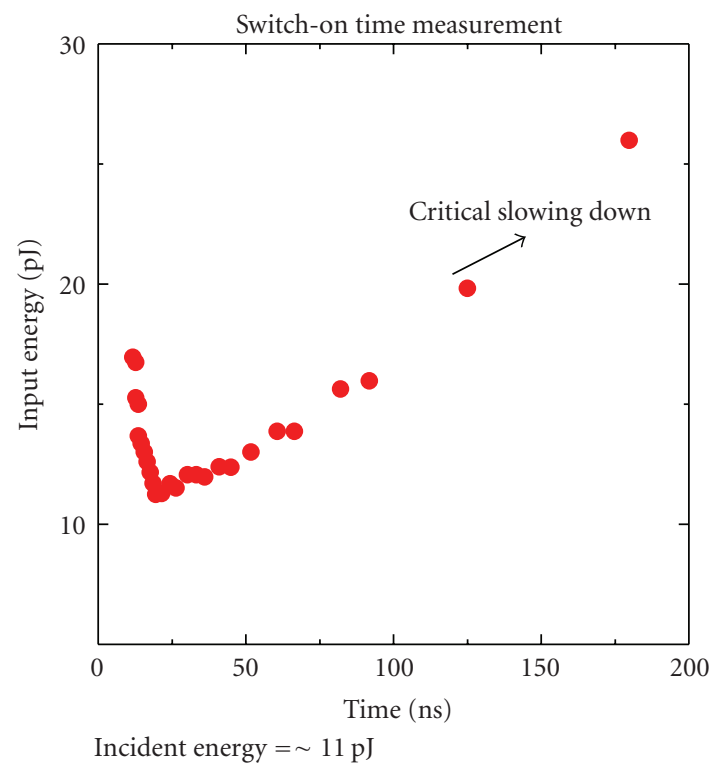

(c)

Figure 3: Temporal response of thermo-optic switching. (a) Switch-off. Temporal response of the probe output. At $t=800$ nanoseconds, the pump signal was switched off. The input instantaneous power for the pump is $64 \mu \mathrm{W}$. The pulse width and period are 400 nanoseconds and 40 microseconds. The exponential fit gives a decay time of approximately 100 nanoseconds. (b) Switch-on operation for various input power. (c) Incident energy required for the switch-on operation estimated from the product of the incident energy and the time required for switch-on in (b).

cavity (the linear absorption of silicon at this wavelength is negligible), and, therefore, we can easily integrate this device with transparent waveguides in the same chip.

Our cavity shown in Figure 2(a) was intentionally designed to have two resonant modes, and, therefore, we can perform all-optical switching using independent two inputs with different wavelengths (signal and control), which is normally required for signal processing. Since bistable switching for one resonant mode should influence the transmission intensity of the other resonant mode in the same cavity, we can easily realize such operation. As we reported in [12], we observe basically similar bistable switching behavior in the relation between the input power for the control mode and the output power for the signal 


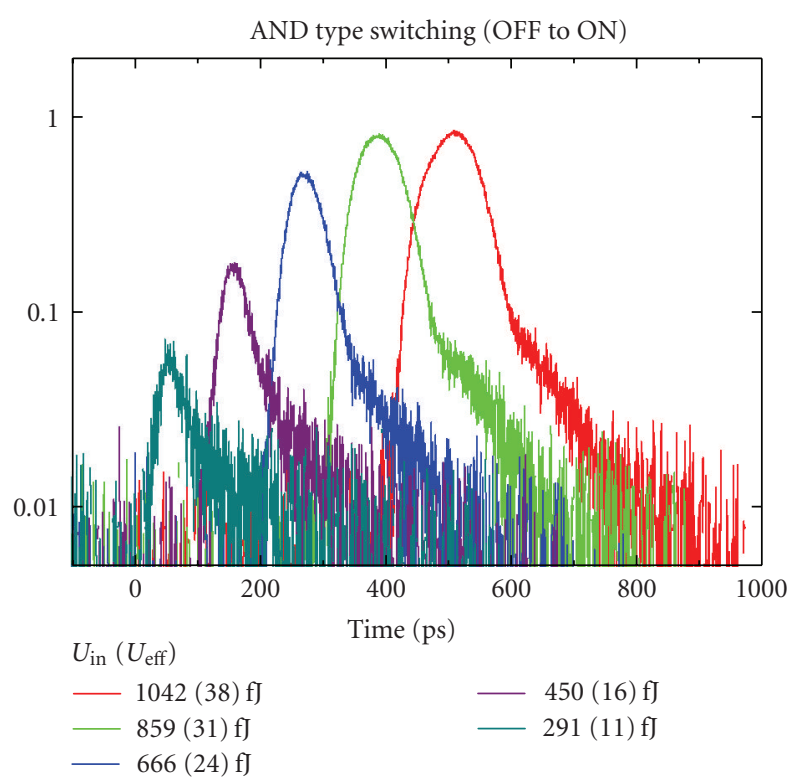

(a)

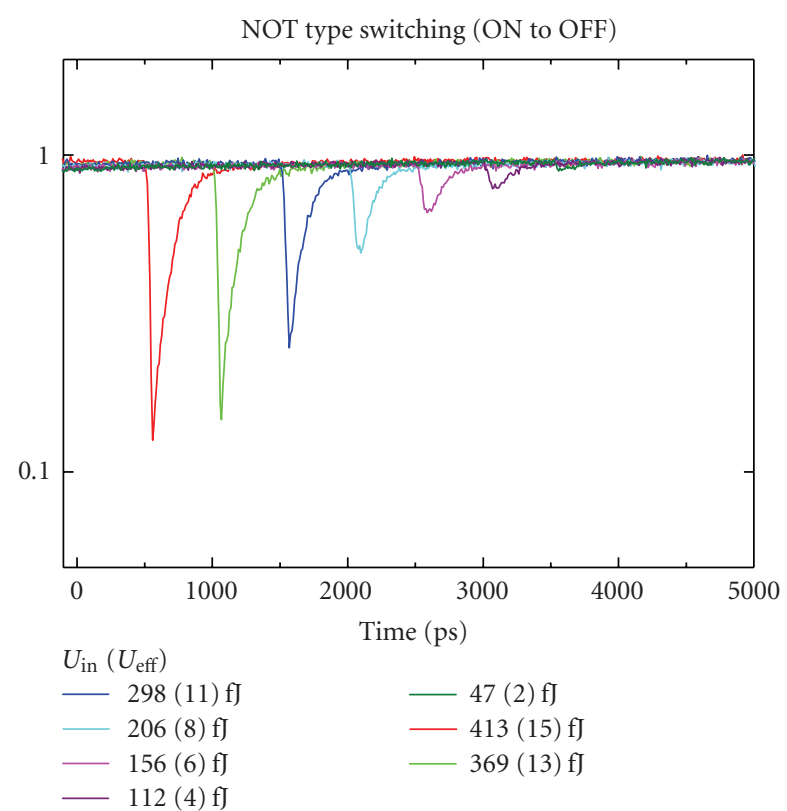

(b)

FIGURE 4: All-optical switching in a silicon photonic crystal nanocavity (end-hole shifted four point cavity) realized by carrier-plasma nonlinearity induced by two-photon absorption in silicon. $Q$ for the control mode is 11500, and $Q$ for the signal mode is 23000 . (a) ANDtype switching at various control pulse energies with the detuning of $-0.3 \mathrm{~nm}$. (b) NOT-type switching at various control pulse energies with no detuning. Each line is shifted by 500 picoseconds.

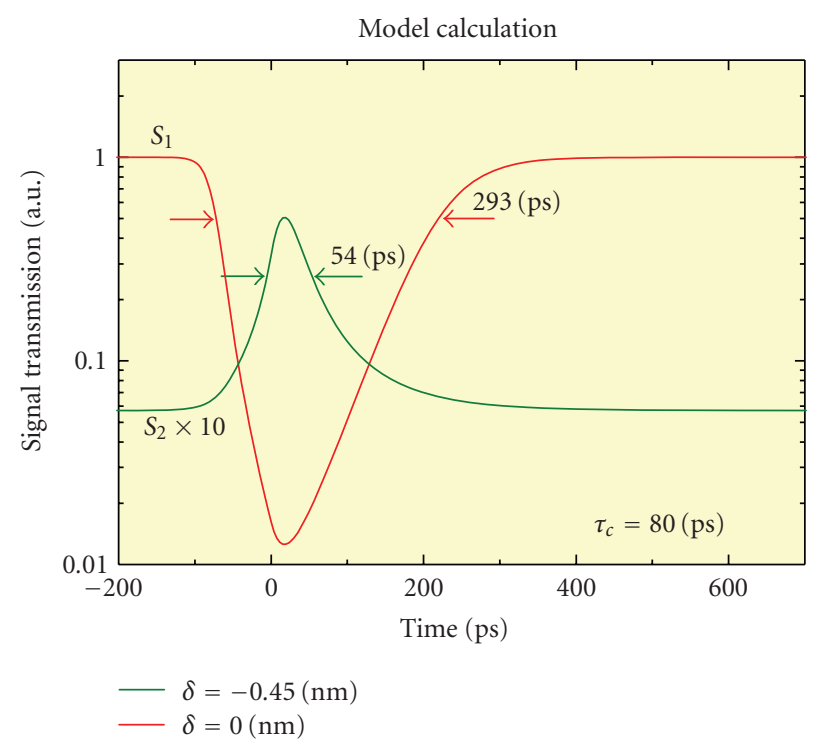

(a)

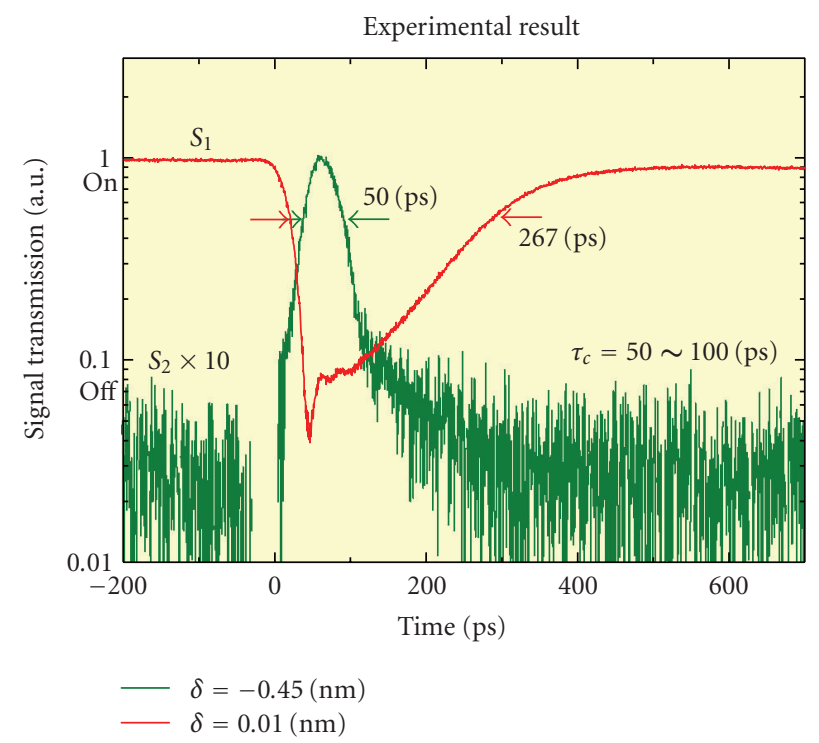

(b)

FIGURE 5: Comparison between the rate-equation analysis and measurement result. We modeled the cavity resonance as a Lorentzian function whose center wavelength is shifting in proportion to the carrier density. (a) Calculation. (b) Experiment.

mode. This operation is basically what we expect for the socalled all-optical transistors, and will be basis for various logic functions. The detail of this operation is described in [12]. For example, we demonstrated that we can amplify an AC signal using this device.
Although the bistable operation itself is similar to that of nonlinear etalon switches, these photonic crystal switches can be clearly distinguished in terms of the operating power and capability for integration. The mode volume of this cavity is only approximately $0.1 \mu \mathrm{m}^{3}$. This small footprint 
itself is of course advantageous for integration, but it is also beneficial for reducing the switching speed because our device is limited by the thermal diffusion process. To demonstrate these characteristics, we measured temporal response of the operation for switch-off and switch-on processes, as shown in Figures 3(a) and 3(b). The relaxation time of our switch is approximately 100 nanoseconds, which is much shorter than that of conventional thermo-optic switches $(\sim$ milliseconds $)$. This amazingly fast thermo-optic switching is primarily due to the smallness of our cavity. In addition, we can estimate the required switching energy from the switch-on measurement. The deduced smallest value is as small as $11 \mathrm{pJ}$ as shown in Figure 3(c).

\section{ALL-OPTICAL SWITCHING BY CARRIER-PLASMA NONLINEARITY}

These thermo-optic nonlinear bistable switches clearly demonstrate that large $Q / V$ photonic crystal cavities are very effective in improving the operation power and speed. However, the speed itself is still not very fast, which is limited by the intrinsically slow thermo-optic effect. To realize much faster all-optical switches, here we employ another nonlinear effect, namely, the carrier-plasma effect [17]. This process is also based on the same TPA process in silicon. Thus most of the arguments concerning their advantages are similar to that for thermo-optic nonlinearity. For this experiment, we used similar photonic crystal cavity devices with a control pulse input. If the duration of the control pulse is sufficiently short, we can avoid thermal heating and may be able to observe only carrier-plasma nonlinearity. In fact, we observed a clear blue shift in the resonance when we injected a 6-picosecond pulse into this device, which is consistent with the expected shift induced by the carrier-plasma nonlinearity. Figure 4 shows the time-resolved output intensity for the signal mode when a 6-picosecond control pulse is input [17]. We observed clear all-optical AND-type switching from OFF to $\mathrm{ON}$ for the detuning of $0.45 \mathrm{~nm}$ (Figure $4(\mathrm{a})$ ) and NOT-type switching from ON to OFF for the detuning of $0.01 \mathrm{~nm}$ (Figure 4(b)). The required switching energy is only a few hundred fJ, which is much smaller than that of ring-cavity-based silicon all-optical switches [18]. If we take the coupling efficiency between the cavity and waveguide into account, the actual pulse energy used for the switching is less than $10 \mathrm{fJ}$. This extremely small switching energy is attributed to large $Q / V$ ratio in our cavity.

The switching time of our device is approximately from 50 picoseconds to 300 picoseconds depending on the detuning and control pulse energy. Considering the fact that the carrier lifetime in silicon is normally very long ( $\sim$ microseconds), this switching time is also surprisingly fast. This apparently indicates that the switching time is not limited by the carrier recombination time of bulk silicon. We analyzed the switching behavior employing simplified rate equations for the photon and carrier density in a photonic-crystal nanocavity incorporating the effective carrier relaxation time. We found that this simple model can reproduce the experimental result fairly well. Figure 5 shows simulated and experimental results for the same parameter condition. In this calculation, we assumed the carrier relaxation time of 80 picoseconds. This suggests that the effective carrier relaxation time for this all-optical switch is in the range between 50 to 100 picoseconds. This fast carrier relaxation may be attributed to the short diffusion time for generated carriers. Note that the photon lifetime of our cavity is approximately 10 picoseconds, and thus the operation speed of our device is limited by the carrier relaxation time. This carrier relaxation time is much shorter than that in other silicon photonic microdevices [18]. That is, the small footprint of the device is again effective in improving the operating speed.

\section{EFFECT OF CARRIER RELAXATION PROCESS ON SWITCHING OPERATION}

In Section 3, we demonstrate that silicon all-optical switches based on carrier-plasma nonlinearity can operate at a significantly fast speed, which is attributed to the fast carrier relaxation process. We regard that this fast carrier relaxation is possibly due to the fact that the diffusion process is an efficient relaxation channel for nanocavities. To verify this explanation, we performed numerical simulations for the carrier diffusion process in silicon photonic crystal cavities [19]. In this simulation, we assumed an initial carrier distribution determined from the optical intensity distribution of the cavity mode, and numerically solved two-dimensional diffusion equations with assuming a realistic photonic crystal cavity structure which is the same as that used in the experiment. The side-wall nonradiative recombination process at the air-hole surface is incorporated in the equation as effective surface recombination rate $(S)$, and the effect of the top-surface nonradiative recombination process is incorporated as an effective carrier lifetime parameter which is determined from another calculation for a simple slab. We calculated the time-dependent resonance wavelength shift using instantaneous carrier concentration in the cavity. The calculated snapshots of the carrier distribution at $t$ $=0,8$, and 24 picoseconds are shown in Figures 6(a), 6(b), and 6(c). These snapshots clearly show that the initial distribution is rapidly spread as a result of diffusion. This rapid diffusion results rapid switching recovery. Figure 6(d) shows the calculated shift of the resonance wavelength for the cavity. The initial wavelength shift caused by the carrier plasma shift is recovered quickly. As shown in the figure, the nonradiative recombination does not seriously affect the initial recovery. Therefore, we believe that the fast operation of our optical switches is explained by the rapid carrier diffusion process.

If we wish to increase the operation speed further, we have to somehow decrease the carrier relaxation time. Although there are several ways to do so, we have recently employed an Ar-ion implantation process in order to introduce extremely fast nonradiative recombination centers into silicon. If the carrier recombination time becomes faster than the diffusion time, we can expect an improvement in the operation speed. When we implanted silicon photonic crystal nanocavity switches with $\mathrm{Ar}^{+}$dose of $2.0 \times 10^{14} \mathrm{~cm}^{-2}$ and an acceleration voltage of $100 \mathrm{keV}$, we observed a significant 


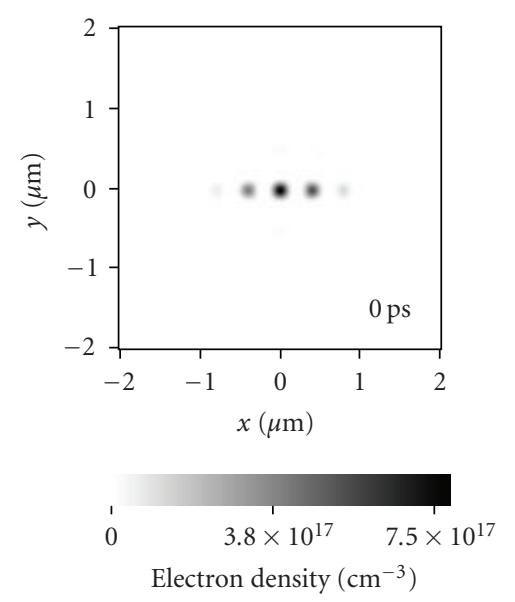

(a)

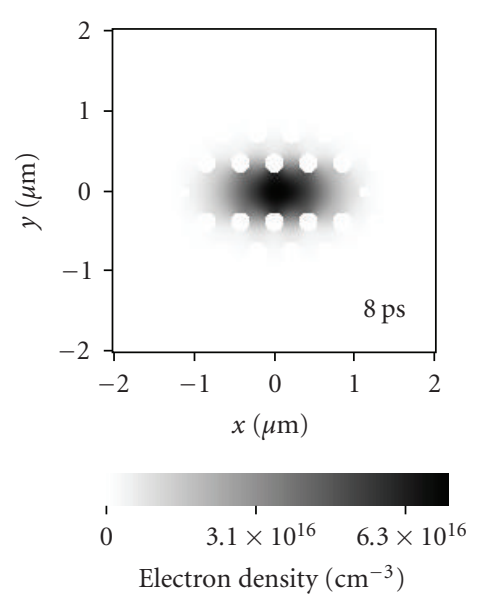

(b)

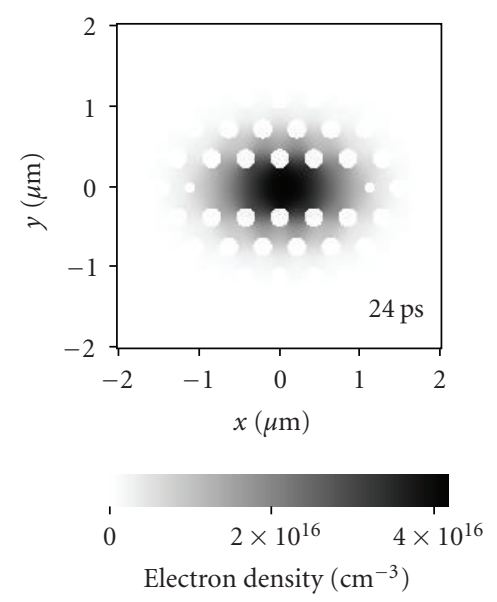

(c)

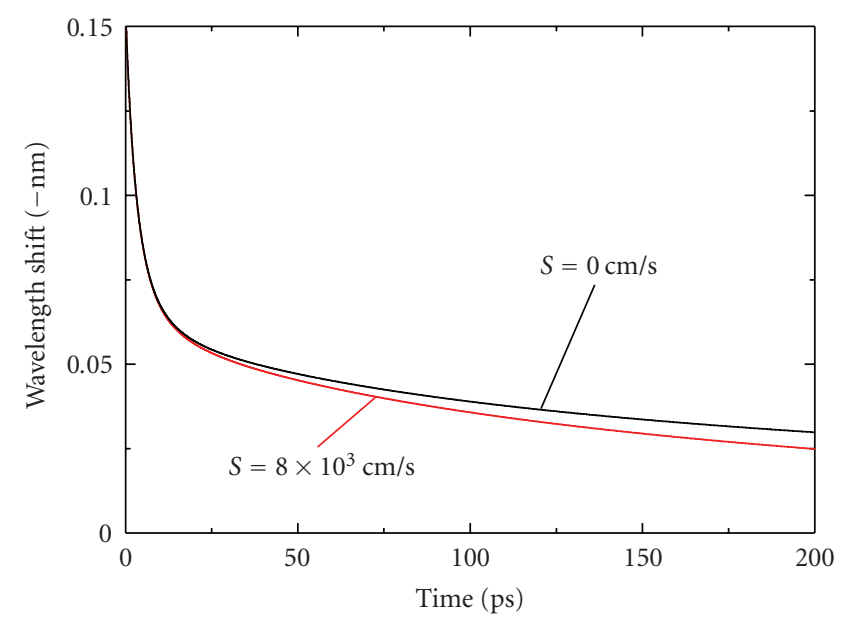

(d)

FIGURE 6: Numerical simulation of carrier diffusion process for a silicon end-hole-shifted photonic crystal cavity. (a), (b), (c) snapshots of the carrier distribution at $t=0,8,24$ picoseconds. (c) Shift of the resonance wavelength for the cavity as a function of time with the sidewall nonradiative recombination rate $S=0$ and $S=8 \times 10^{3} \mathrm{~cm} / \mathrm{s}$.

improvement in switching speed as shown in Figures $7(\mathrm{a})$, 7(b). The implantation condition was carefully determined so as to keep almost the same cavity $Q$ but to significantly decrease the carrier lifetime in the cavity. In the case of detuning for an NOT gate (Figure 7(a), it was reduced from 110 picoseconds to 50 picoseconds. In the case of detuning for an AND gate, the switching time was reduced from 220 picoseconds to 70 picoseconds (Figure 7(b)) [20]. As shown in Figure $7(\mathrm{c})$, the ON/OFF ratio is mostly the same between two conditions. In addition, the required switching energy was almost the same as that without the implantation. Although the reduction of the carrier lifetime may lead to increase in the switching energy, this change is not significant as far as the carrier lifetime is longer than the photon lifetime and the control pulse length (which was the case in our experiment). These results clearly demonstrate the effect of ion-implantation on the improvement in the switching speed.

\section{ALL-OPTICAL MEMORY OPERATION AND LOGIC CIRCUIT}

In the same way as thermo-optic switching, carrier-plasma switching also provides bistable operation. Figure 8 shows bistable operations realized by employing a pair of set and reset pulses [21]. When a set pulse is fed into the input waveguide, the output signal is switched from OFF to ON and remains $\mathrm{ON}$ even after the set pulse exits (green curve). When a pair of set and reset pulses is applied (as shown in Figure $8(\mathrm{a})$ ), the output is switched from OFF to $\mathrm{ON}$ by the set pulse and then ON to OFF by the reset pulse (blue curve). This is simply a memory operation using optical bistability. The energy of the set pulse is less than $100 \mathrm{fJ}$, and the DC bias input for sustaining the ON/OFF states is only $0.4 \mathrm{~mW}$. These small values are primarily the results of the large $Q / V$ ratio of the photonic crystal cavity. It is worth noting that the largest $\mathrm{Q} / V$ should always result in the smallest switching power, 


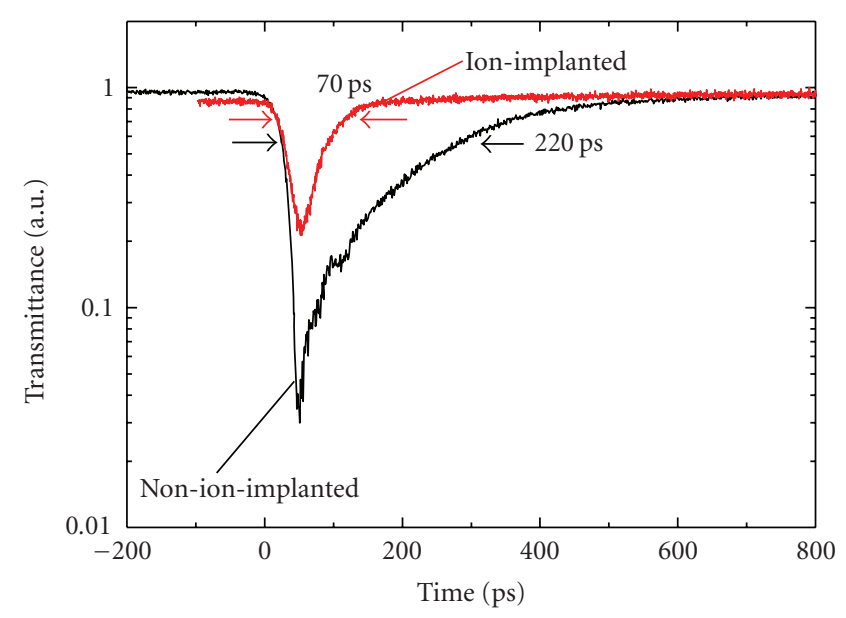

(a)

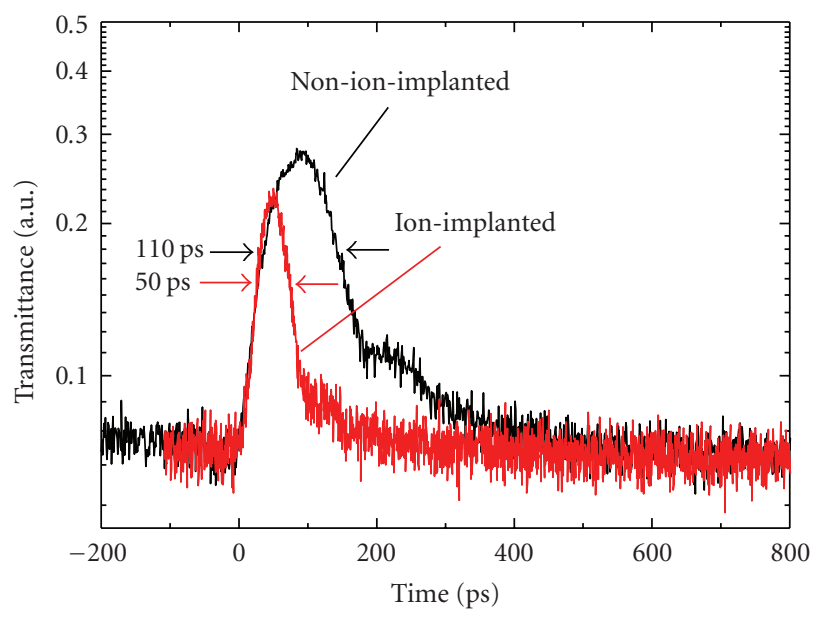

(b)

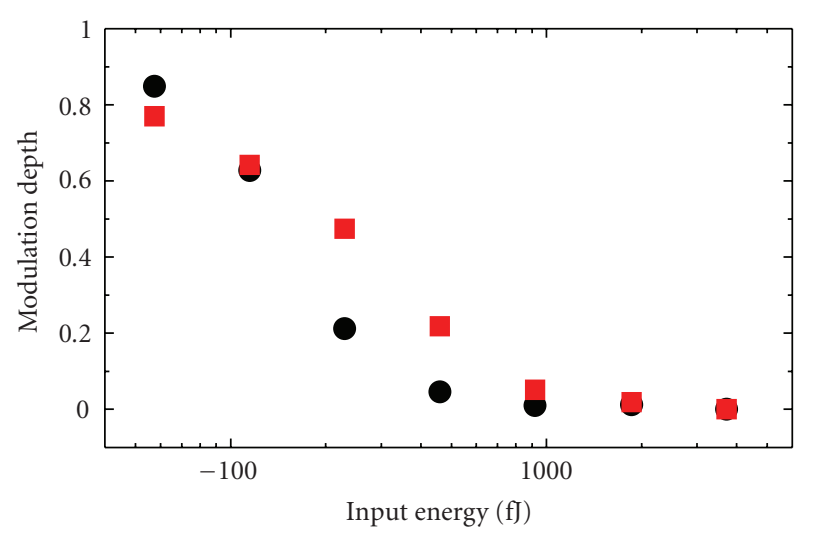

- Non-ion-implanted

Ion-implanted

(c)

FIGURE 7: All-optical switching for samples with and without ionimplantation. (a) NOT-type switching. (b) AND-type switching. (c) Signal transmission at the time when the control pulses enters the cavity. The ON/OFF contrast of the signal is shown in relation to the input control pulse energy. The circles are the minimal transmission for the nonimplanted sample, and the squares are for the implanted sample. but the operation speed can be limited by $Q$. In the present situation, the switching speed is still limited by the carrier relaxation time, and thus a large $Q / V$ is preferable. In the case when the photon lifetime limits the operation speed, we have to choose appropriate loaded $Q$ for the required speed. Even in such a case, it is better to have high unloaded $Q$ because loaded $Q$ can be controlled by changing the cavitywaveguide coupling, and high unloaded $Q$ means low loss of the device. The best design of out device would be a device with the smallest volume, the lowest transmission loss, and the designated loaded $Q$ (depending on the operation speed). The lowest loss with the designated loaded $Q$ can be obtained only when we employs an ultrahigh unloaded $Q$ cavity. Compared with other types of all-optical memories, this device has several advantages, such as small footprint, low-energy consumption, and the capability for integration. The fact that all the light signals used for the operation are transparent in waveguides is important for the application, which is fundamentally different from bistable-laser-based optical memories.

In the above, we showed that a single photonic crystal cavity coupled to waveguides functions as a bistable switch or a memory. If we couple two or more bistable cavities, we can create much more complex logic functions [11], in the same way as with transistor-based logic in electronics. As an example, here we show our numerical design for an all-optical flip-flop consisting of two bistable cavities integrated in a photonic crystal. It has been proposed that alloptical flip-flops be realized by using two nonlinear etalons with appropriate cross-feedback [22], but this proposal is unsuitable for on-chip integration. Here, we propose a different design using two photonic crystal nanocavities [23].

A typical example of flip-flop operation in the highspeed information processing is a retiming circuit, which corrects the timing jitter of an information bitstream and synchronizes it with the clock pulses. This function is normally accomplished by high-speed electronic circuits, but if it can be done all optically, it will be advantageous for future ultrahigh-speed data transmission. Here, we propose a simple flip-flop design for realizing the retiming function. Figure 9(a) shows a design for the retiming circuit. The coupled cavities (C1 and C2) have one common resonant mode $\left(\lambda_{2}=1548.48 \mathrm{~nm}, Q_{2}=4500\right)$ extended to both cavities and two modes $\left(\lambda_{1}=1493.73 \mathrm{~nm}, Q_{1}=6100\right.$, and $\left.\lambda_{3}=1463.46 \mathrm{~nm}, Q_{3}=4100\right)$ localized in each cavity. Here, we use two bistable switching operations for $\mathrm{C} 1$ and C2. The cross-feedback is realized as follows. C1 is switched ON only when $\lambda_{1}$ and $\lambda_{2}$ are both applied ( $\mathrm{P}_{\mathrm{IN}} 1$ and $\mathrm{P}_{\mathrm{IN}} 2$ are $\mathrm{ON}) . \mathrm{C} 2$ is $\mathrm{ON}$ only when $\lambda_{3}$ are applied $\left(\mathrm{P}_{\mathrm{IN}} 3\right.$ is $\left.\mathrm{ON}\right)$ and simultaneously $\lambda_{2}$ is supplied from $\mathrm{C} 1$ (which means $\mathrm{C} 1$ is $\mathrm{ON}$ ). Thus the output signal of $\lambda_{3}$ ( $\left.\mathrm{P}_{\mathrm{OUT}} 3\right)$ becomes $\mathrm{ON}$ only if $\mathrm{P}_{\mathrm{IN}} 3$ is turned $\mathrm{ON}$ when $\mathrm{C} 1$ is already $\mathrm{ON}$ in advance. These results achieve retiming process. We set $\mathrm{P}_{\mathrm{IN}} 1$ and $\mathrm{P}_{\mathrm{IN}} 3$ as two different clock signals as shown in Figure 9(b), and assume $\mathrm{P}_{\mathrm{IN}} 2$ to be bit-stream NRZ (nonreturn-to-zero) data with finite timing jitter. The resultant $\mathrm{P}_{\text {OUT }} 3$ is precisely synchronized to the clock signals and is actually an RZ (return-to-zero) data stream converted from $\mathrm{P}_{\mathrm{IN}} 2$ with jitter corrected. 


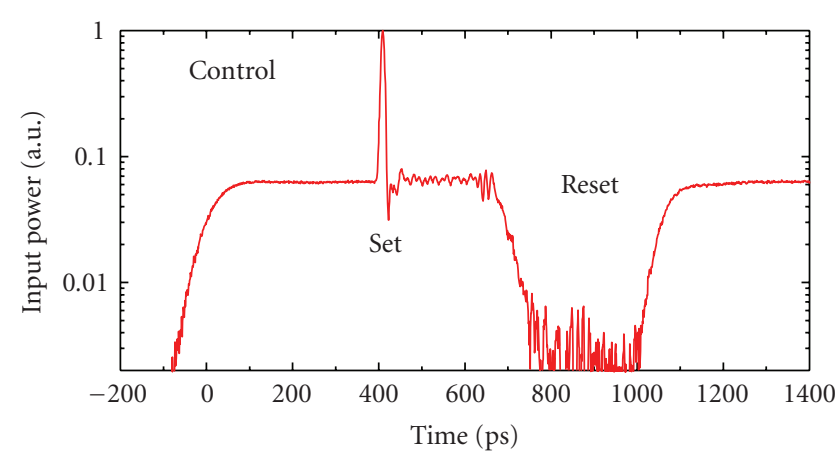

(a)

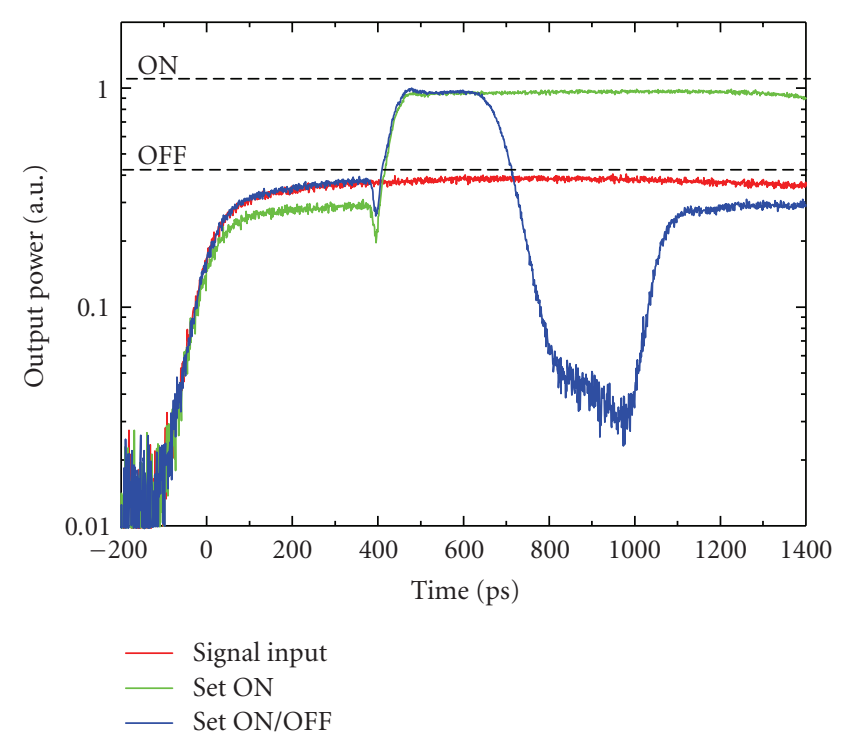

(b)

FIgURE 8: All-optical bistable memory operation in a silicon photonic crystal nanocavity (end-hole shifted four-point defect cavity) realized by the carrier-plasma nonlinearity induced by twophoton absorption in silicon. $Q$ for the control mode is 7640, and $Q$ for the signal mode is 12400 . (a) Injected control light consisting of a pair of set and reset pulses. (b) Output signal intensity as a function of time for three different cases: with no set/reset pulses (red curve), with set pulse only (green curve), and with set and reset pulses (blue curve).

We designed this function in a photonic crystal slab system, and numerically simulated its operation using the FDTD method. The structural parameters are shown in the figure caption. We assumed realistic material parameters (with a Kerr coefficient $\chi^{(3)} / \mathcal{E}_{0}=4.1 \times 10^{-19}\left(\mathrm{~m}^{2} / \mathrm{V}^{2}\right)$, a typical value for $\mathrm{AlGaAs}$ ) and the instantaneous driving power is assumed to be $60 \mathrm{~mW}$ for all three inputs. Figure 9(b) shows three input signals (a data stream with jitter, and two clock pulses), and the output from $\mathrm{P}_{\mathrm{D}}\left(\mathrm{P}_{\mathrm{OUT}} 3\right)$. As seen in this plot, $\mathrm{P}_{\text {OUT }} 3$ is the RZ signal of the input with the jitter corrected. We confirmed that the operation speed corresponds to $50 \mathrm{GHz}$ operation. Note that this work was intended to demonstrate the operation principle and the structure has not yet been optimized. We expect $\sim \mathrm{mW}$

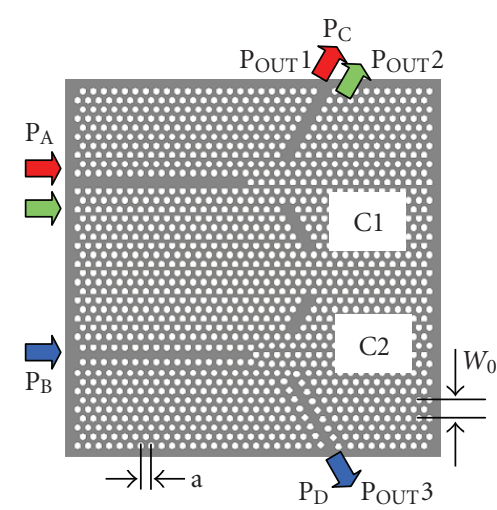

(a)

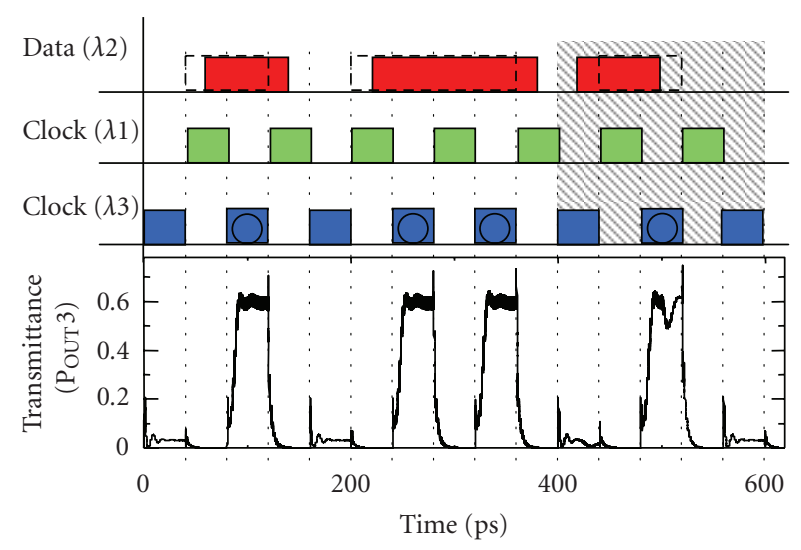

(b)

FIGURE 9: All-optical retiming circuit based on two bistable cavities. (a) Design based on a hexagonal air-hole 2D photonic crystal with $a=400 \mathrm{~nm}$ and $2 r=0.55 a$. Two waveguides in the upper area $\left(\mathrm{P}_{\mathrm{A}}\right.$ and $\left.\mathrm{P}_{\mathrm{C}}\right)$ are $\mathrm{W} 1$ and the other two in the lower area $\left(\mathrm{P}_{\mathrm{B}}\right.$ and $\left.\mathrm{P}_{\mathrm{D}}\right)$ are W0.8. (b) Simulated operation.

operation may be possible after the optimization since our rough estimation shows that a single cavity switch can operate at sub-mW input power for the similar condition. In addition, if we manage to employ the carrier-plasma effect for this operation (as was done in Figure 8), we can expect further decrease in terms of the input power.

In addition to this work, we have reported another design of all-optical logic circuit which is mostly equivalent to an SR flip-flop employing two bistable cavities [8].

\section{SUMMARY}

Recent rapid progress in photonic crystal nanocavities is enabling low-power, all-optical switching and memory actions on a silicon chip. We have shown that our experimental demonstration of all-optical switching operation using thermo-optic nonlinearity and carrier-plasma nonlinearity, both based on two-photon absorption in silicon. For both cases, we observed significant decrease in the switching power (energy) and also significant increase in the switching speed. In this article, we numerically investigated the 
diffusion process concerning photonic-crystal nanocavities, which is fundamentally different from that in conventional optical devices with much larger size. Moreover, we have demonstrated their potential for optical logic by a combination of bistable elements. For the application in alloptical processing, a photonic crystal has several important advantages, such as small footprint, small energy consumption, high speed, and significant integratability. Thus we believe that it may be a promising candidate for all-optical information processing on a silicon chip.

\section{REFERENCES}

[1] B.-S. Song, S. Noda, T. Asano, and Y. Akahane, "Ultrahigh-Q photonic double-heterostructure nanocavity," Nature Materials, vol. 4, no. 3, pp. 207-210, 2005.

[2] S. Noda, M. Fujita, and T. Asano, "Spontaneous-emission control by photonic crystals and nanocavities," Nature Photonics, vol. 1, no. 8, pp. 449-458, 2007.

[3] E. Kuramochi, M. Notomi, S. Mitsugi, A. Shinya, T. Tanabe, and T. Watanabe, "Ultrahigh-Q photonic crystal nanocavities realized by the local width modulation of a line defect," Applied Physics Letters, vol. 88, no. 4, Article ID 041112, 3 pages, 2006.

[4] T. Tanabe, M. Notomi, E. Kuramochi, A. Shinya, and H. Taniyama, "Trapping and delaying photons for one nanosecond in an ultrasmall high-Q photonic-crystal nanocavity," Nature Photonics, vol. 1, no. 1, pp. 49-52, 2007.

[5] R. Herrmann, T. Sünner, T. Hein, A. Löffler, M. Kamp, and A. Forchel, "Ultrahigh-quality photonic crystal cavity in GaAs," Optics Letters, vol. 31, no. 9, pp. 1229-1231, 2006.

[6] T. Tanabe, A. Shinya, E. Kuramochi, S. Kondo, H. Taniyama, and M. Notomi, "Single point defect photonic crystal nanocavity with ultrahigh quality factor achieved by using hexapole mode," Applied Physics Letters, vol. 91, no. 2, Article ID 021110, 3 pages, 2007.

[7] T. Tanabe, M. Notomi, E. Kuramochi, and H. Taniyama, "Large pulse delay and small group velocity achieved using ultrahigh-Q photonic crystal nanocavities," Optics Express, vol. 15, no. 12, pp. 7826-7839, 2007.

[8] M. Notomi, T. Tanabe, A. Shinya, et al., "Nonlinear and adiabatic control of high-Q photonic crystal nanocavities," Optics Express, vol. 15, no. 26, pp. 17458-17481, 2007.

[9] M. Soljačić and J. D. Joannopoulos, "Enhancement of nonlinear effects using photonic crystals," Nature Materials, vol. 3, no. 4, pp. 211-219, 2004.

[10] H. M. Gibbs, Optical Bistability: Controlling Light with Light, Academic Press, Orlando, Fla, USA, 1985.

[11] S. D. Smith, "Optical bistability, photonic logic, and optical computation," Applied Optics, vol. 25, no. 10, pp. 1550-1564, 1986.

[12] M. Notomi, A. Shinya, S. Mitsugi, G. Kira, E. Kuramochi, and T. Tanabe, "Optical bistable switching action of Si highQ photonic-crystal nanocavities," Optics Express, vol. 13, no. 7, pp. 2678-2687, 2005.

[13] S. Mitsugi, A. Shinya, E. Kuramochi, M. Notomi, T. Tshchizawa, and T. Watanabe, "Resonant tunneling wavelength filters with high $Q$ and high transmittance based on photonic crystal slabs," in Proceedings of the 16th Annual Meeting of IEEE Lasers and Electro-Optics Society (LEOS '03), vol. 1, pp. 214-215, Tucson, Ariz, USA, October 2003.

[14] M. Notomi, A. Shinya, S. Mitsugi, E. Kuramochi, and H.-Y. Ryu, "Waveguides, resonators and their coupled elements in photonic crystal slabs," Optics Express, vol. 12, no. 8, pp. 1551$1561,2004$.

[15] G. R. Olbright, N. Peyghambarian, H. M. Gibbs, H. A. MacLeod, and F. Van Milligen, "Microsecond roomtemperature optical bistability and crosstalk studies in $\mathrm{ZnS}$ and ZnSe interference filters with visible light and milliwatt powers," Applied Physics Letters, vol. 45, no. 10, pp. 1031-1033, 1984.

[16] V. R. Almeida and M. Lipson, "Optical bistability on a silicon chip," Optics Letters, vol. 29, no. 20, pp. 2387-2389, 2004.

[17] T. Tanabe, M. Notomi, S. Mitsugi, A. Shinya, and E. Kuramochi, "All-optical switches on a silicon chip realized using photonic crystal nanocavities," Applied Physics Letters, vol. 87, no. 15, Article ID 151112, 3 pages, 2005.

[18] V. R. Almeida, C. A. Barrios, R. R. Panepucci, and M. Lipson, "All-optical control of light on a silicon chip," Nature, vol. 431, no. 7012, pp. 1081-1084, 2004.

[19] T. Tanabe, H. Taniyama, and M. Notomi, "Carrier diffusion and recombination in photonic crystal nanocavity optical switches," Journal of Lightwave Technology, vol. 26, no. 11, pp. 1396-1403, 2008.

[20] T. Tanabe, K. Nishiguchi, A. Shinya, et al., "Fast alloptical switching using ion-implanted silicon photonic crystal nanocavities," Applied Physics Letters, vol. 90, no. 3, Article ID 031115, 3 pages, 2007.

[21] T. Tanabe, M. Notomi, S. Mitsugi, A. Shinya, and E. Kuramochi, "Fast bistable all-optical switch and memory on a silicon photonic crystal on-chip," Optics Letters, vol. 30, no. 19, pp. 2575-2577, 2005.

[22] H. Tsuda and T. Kurokawa, "Construction of an all-optical flip-flop by combination of two optical triodes," Applied Physics Letters, vol. 57, no. 17, pp. 1724-1726, 1990.

[23] A. Shinya, S. Mitsugi, T. Tanabe, et al., "All-optical flip-flop circuit composed of coupled two-port resonant tunneling filter in two-dimensional photonic crystal slab," Optics Express, vol. 14, no. 3, pp. 1230-1235, 2006. 

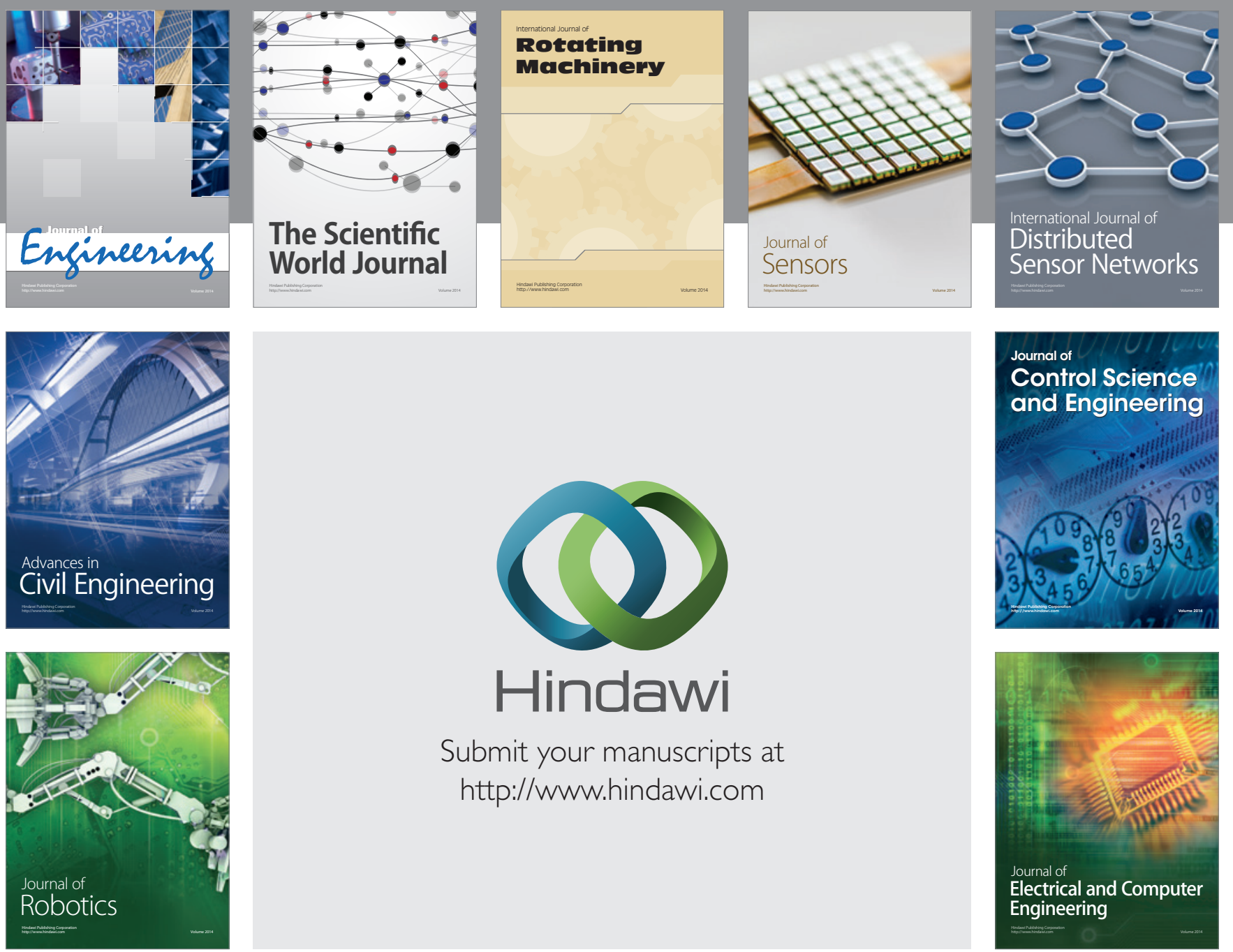

Submit your manuscripts at

http://www.hindawi.com
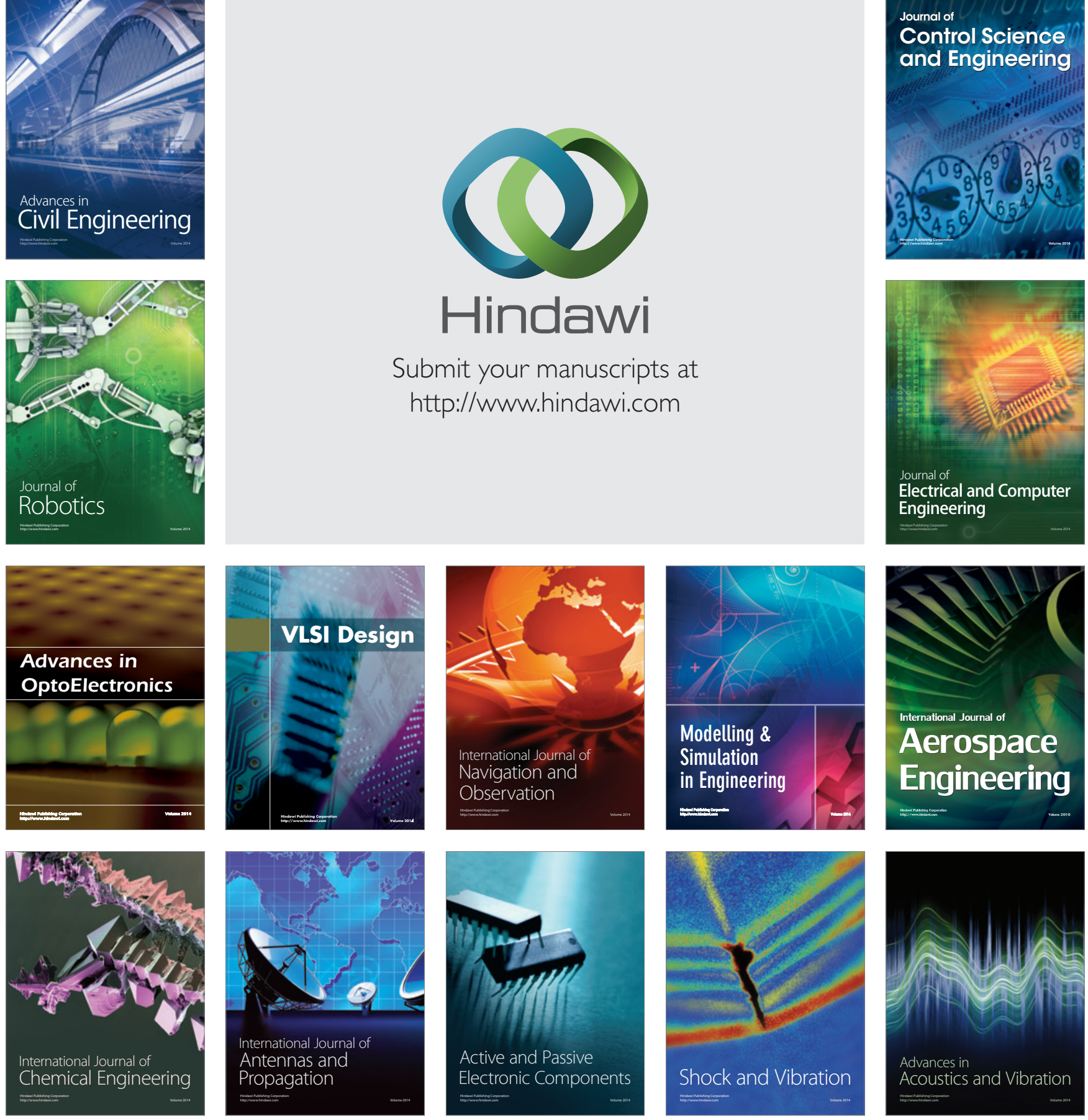